\title{
Un aperçu des rythmes scolaires dans le monde
}

\section{Bernadette Plumelle}

\section{OpenEdition}

Journals

Édition électronique

URL : https://journals.openedition.org/ries/2045

DOI : $10.4000 /$ ries. 2045

ISSN : 2261-4265

\section{Éditeur}

France Education international

\section{Édition imprimée}

Date de publication : 1 septembre 2011

Pagination : 16-20

ISBN : 978-2-85420-592-3

ISSN : $1254-4590$

\section{Référence électronique}

Bernadette Plumelle, «Un aperçu des rythmes scolaires dans le monde », Revue internationale d'éducation de Sèvres [En ligne], 57 | septembre 2011, mis en ligne le 01 septembre 2014, consulté le 06 juillet 2021. URL : http://journals.openedition.org/ries/2045 ; DOI : https://doi.org/10.4000/ries. 2045

Ce document a été généré automatiquement le 6 juillet 2021.

(ㄷ) Tous droits réservés 


\title{
Un aperçu des rythmes scolaires dans le monde
}

\author{
Bernadette Plumelle
}

1 Au mois de mars 2010, Luc Chatel, ministre de l'Éducation nationale, de la Jeunesse et de la Vie associative, annonçait la création en France d'un débat de grande ampleur sur la question des rythmes scolaires. Avec la Conférence des rythmes scolaires ${ }^{1}$ mise en place le 7 juin 2010, des dizaines d'auditions et des centaines de réunions ont été organisées et un site public a offert une plate-forme de discussion et d'échanges afin que tous les sujets soient discutés : le rythme pendant la journée, pendant la semaine et pendant l'année. Les comparaisons internationales, des descriptions de l'organisation du temps scolaire dans d'autres pays et des témoignages ${ }^{2}$ ont également alimenté les réflexions. Quelques mois auparavant; l'OCDE et Eurydice avaient, dans deux rapports, produits des études comparatistes sur ce sujet. L'ensemble des informations recueillies permet de dessiner un paysage très éclaté des rythmes scolaires.

\section{Sur quoi porte la comparaison?}

Le rapport d'Eurydice "Chiffres clés de l'éducation en Europe 2009 » offre une comparaison portant sur le temps annuel d'enseignement de trente-et-un pays. Dans son dernier rapport annuel Regards sur l'éducation 2010, l'OCDE propose un ensemble d'indicateurs destiné à analyser «l'environnement pédagogique et l'organisation scolaire » dans les pays membres et partenaires de l'OCDE. L'indicateur relatif au temps d'instruction évalue le temps que les élèves âgés de 7 à 15 ans doivent en principe passer en classe et la répartition de ce temps entre les différentes matières. Ces deux études révèlent une variété très grande des rythmes scolaires avec trois facteurs pris en compte: l'organisation annuelle du temps scolaire; les rythmes hebdomadaires et l'aménagement de la journée. Le mode de gouvernance des systèmes éducatifs est déterminant en la matière. En effet, dans de nombreux pays, les établissements ou les autorités locales chargées de l'éducation sont libres de décider du nombre d'heures de cours à dispenser et de leur répartition entre les diverses matières. 


\section{Organisation des rythmes scolaires : qui décide?}

Les niveaux de décision sont très différents selon que les pays ont un système éducatif de type centralisé, décentralisé ou fédéral. Dans l'ensemble, la durée de l'année scolaire est décidée en général au niveau national (nombre de semaines, de jours ou d'heures), de même que la date de rentrée et de sortie administrative. Mais les dates de rentrée et de fin scolaire sont souvent laissées à l'appréciation des régions, voire des établissements scolaires. Et s'il y des obligations nationales, elles portent sur le nombre d'heures de cours par jour ou l'heure limite de sortie. Ainsi le calendrier scolaire est décidé au niveau national au Portugal ou en République tchèque. En Allemagne, il est fixé au niveau fédéral mais les dates des " petites vacances " sont décidées au niveau du Land. En Espagne, chaque communauté autonome établit le calendrier scolaire. En Angleterre, même s'il y a des indications nationales, les dates de congés dépendent de l'autorité régionale et les écoles privées décident en toute indépendance de la rentrée et sortie des classes. En Suède, les dates de début et de fin d'année scolaire sont décidées au niveau de la commune ou par le Conseil regroupant les établissements indépendants. En Finlande ou en Corée du Sud, le chef d'établissement a autorité pour fixer les dates des vacances scolaires et de la rentrée des classes.

\section{Forte variabilité de la durée de l'année scolaire}

4 En moyenne, dans les pays de l'OCDE, le temps total d'enseignement prévu représente en moyenne 6777 heures entre 7 et 14 ans. Mais il varie selon les pays; il s'établit à 4715 heures en Pologne mais dépasse 8000 heures en Italie. La France est l'un des pays où le nombre d'heures est le plus important (7 773 heures); seuls quelques pays ont un nombre d'heures d'instruction plus important: l'Australie, les Pays-Bas, l'Italie et Israël. À l'inverse, en France le nombre de semaines d'enseignement est peu élevé, 35 semaines, alors qu'au Danemark, il est de 42 semaines. La moyenne des pays se situe autour de 37 à 38 semaines.

Le temps scolaire est rythmé par des vacances estivales et des vacances intermédiaires, qui scandent l'année scolaire. Mais ce temps scolaire s'organise de manière variable. Les vacances estivales peuvent aller du simple au double. En Europe voici quelques cas de figure : en Allemagne ou au Danemark, elles sont de six semaines; en France elles sont de neuf semaines, de dix semaines en Finlande ou en Italie et jusqu'à douze semaines en Espagne. Ces vacances sont en moyenne plus longues en Europe que sur d'autres continents. Au Canada, leur durée est de huit semaines ; au Brésil, elles sont de six semaines. Enfin, en Asie, les vacances ont une durée de quatre à cinq semaines (Japon ou Corée du sud).

\section{Rythmes hebdomadaires et amplitude horaire de la journée}

6 Dans la majorité des pays, la semaine d'enseignement est de cinq jours. Pour quelques pays, l'amplitude hebdomadaire est de six jours. En Corée du Sud, les semaines vont du lundi au samedi inclus, sachant que deux samedi par mois doivent être libres. C'est 
également le cas pour quelques Länder en Allemagne. En Angleterre, il existe parfois des activités optionnelles le samedi. En Italie, les cours doivent être répartis au moins sur cinq jours par semaine mais il est très fréquent, particulièrement dans le premier cycle, que les cours aient lieu du lundi au samedi inclus. En Pologne, les écoles ont la possibilité de proposer des cours le samedi matin, de manière ponctuelle ou régulière (après consultation des parents et des enseignants). Cette organisation peut être différente pour les établissements privés. Au Japon, par exemple, les samedis et dimanches sont des jours sans école (arrêté ministériel) mais certaines écoles privées organisent des cours le samedi. La France constitue une exception, avec la semaine de quatre jours dans le cycle primaire.

7 Dans le secteur primaire, la moyenne de l'OCDE est de 4,1 heures/jour et celle de l'Europe (19 pays) est de 4,2. Le Chili offre une moyenne de 5,7 heures de cours journalier avec, à l'autre extrême, la Russie, la Hongrie ou la Corée du Sud qui ont une durée quotidienne de trois heures de cours par jour. En France, la journée est de six heures (sur quatre jours).

Le calcul pour l'enseignement secondaire inférieur (premier cycle) se fait sur une base hebdomadaire. La moyenne de l'OCDE est de 23,5 heures par semaine et celle de l'Europe (19 pays) de 23,4 avec des écarts importants entre le Mexique (27,8 heures de cours hebdomadaires) et la Hongrie (18,1 heures). La France a un des taux les plus élevés, avec 27,6 heures de cours hebdomadaires.

La durée journalière diffère selon que la semaine est de cinq ou six jours. Dans le premier cas, la journée commence en moyenne entre $8 \mathrm{~h}$ et $9 \mathrm{~h}$ du matin et se termine vers $15 \mathrm{~h}$ ou $16 \mathrm{~h}$. En Angleterre, la journée débute généralement aux alentours de $9 \mathrm{~h}$ et se termine vers $15 \mathrm{~h}$ pour l'école primaire et $15 \mathrm{~h} 30$ pour le secondaire. Un nouveau système, "extended school day ", vient d'être institué, où les élèves peuvent suivre des cours optionnels avant ou après l'emploi du temps normal.

En Italie, les élèves ont cours de $8 \mathrm{~h}$ à $13 \mathrm{~h}$ pour les semaines à six jours. Sur cinq jours, les cours ont lieu de $8 \mathrm{~h}$ à $16 \mathrm{~h}$. Cette variation se retrouve en Allemagne. Les élèves ont cours de $7 \mathrm{~h} 30$ ou $8 \mathrm{~h} 30$ à $11 \mathrm{~h} 30$ sur six jours et de $7 \mathrm{~h} 30$ ou $8 \mathrm{~h} 30$ à $13 \mathrm{~h}$ ou $14 \mathrm{~h}$ pour les semaines à cinq jours.

\section{Un cadre législatif variable selon les pays}

11 En Finlande, une journée d'école ne peut excéder cinq séquences pour les deux premières années d'enseignement obligatoire. Par la suite, le nombre maximum de séquences par jour est fixé à sept. Les élèves du cycle primaire terminent leur journée scolaire vers $13 \mathrm{~h}$, ceux du collège terminent à $14 \mathrm{~h}$. En Angleterre, la loi fixe uniquement le minimum d'heures de cours par semaine et par niveau d'enseignement : de 21 heures/semaine pour un élève âgé de cinq ans à 25 heures de cours pour un élève de 16 ans ; elle ne donne aucune indication en termes de nombre maximal de cours par jour ou par semaine. Au Danemark, pour les trois premières années du primaire, la journée ne peut aller au-delà de sept heures d'enseignement. En Suède, le nombre d'heures de cours par jour est imposé par une législation nationale. En Ontario, une journée de classe ne peut commencer avant $8 \mathrm{~h}$ ni se terminer après $17 \mathrm{~h}$ sans l'accord du ministre. 


\section{Comment s'organise la journée d'école?}

12 La durée d'une séquence d'enseignement s'échelonne entre 30 et 90 minutes et peut varier, pour un même pays, selon le cycle d'enseignement. Dans quelques pays, les établissements ont toute latitude pour « fractionner » la journée. Pour une majorité des pays étudiés, la séquence d'enseignement est de 45 minutes comme, par exemple, en Allemagne, au Brésil, au Danemark, en Pologne ou en République tchèque. La durée d'un cours peut différer selon le niveau d'enseignement, primaire ou secondaire. En Finlande, pour les niveaux primaire et secondaire inférieur (collège), le cours est de 45 minutes; il est de $1 \mathrm{~h} 15$ au secondaire supérieur (lycée). En Espagne, la durée d'un cours varie entre 45 minutes et 60 minutes pour les premiers niveaux. Il est de 60 minutes pour les élèves de lycée.

Dans quelques pays, aucune règle n'est imposée. En Angleterre, chaque établissement est libre de son organisation. En Écosse, il n'y a pas de durée fixe pour un cours. En règle générale, les séquences éducatives durent entre 55 et 60 minutes. En Suède, les rythmes quotidiens sont décidés au niveau de l'établissement.

\section{Réformes et débats au sujet des rythmes scolaires}

14 Le rapport annuel Regards sur l'éducation 2010 de l'OCDE récapitule, dans un tableau, les réformes concernant le temps d'instruction annuel mises en œuvre entre 1995 et 2010 pour quinze pays dont les données étaient disponibles. Une majorité des pays a diminué le temps d'instruction: l'Autriche, la Belgique (Communauté flamande), la France, la Hongrie, l'Italie et les Pays-Bas. À l'inverse, quelques pays ont augmenté le temps d'instruction (Finlande, Norvège, Portugal). Mais l'Allemagne est le pays dont l'organisation du temps scolaire a été profondément modifiée à la suite de réformes. Les mauvaises performances à l'enquête PISA (2000, 2003).ont amené l'Allemagne à reconsidérer l'organisation des rythmes scolaires et à proposer un allongement de la journée scolaire. Deux aménagements du temps scolaire ont été mis en place; le développement d'écoles à temps plein ${ }^{3}$ en remplacement de la journée continue, source d'inégalités scolaires, et la réduction d'une année de l'enseignement secondaire (réforme «G 8 »). Un programme d'investissement, Zukunft Bildung und Betreuung, a permis à de nombreux établissements d'offrir un service d'accueil et des activités tout au long de la journée, les Ganztagsschulen (" écoles ouvertes toute la journée »). Dans certains Länder, la scolarité a été réduite de 9 à 8 ans, avec pour conséquence une augmentation du nombre d'heures par semaine. Dans les Gymnasium (lycée) en huit ans, cette augmentation du nombre d'heures de cours a provoqué des débats parmi les parents et les élèves, à cause de la surcharge de travail.

Le système de la "journée continue » fait également débat dans d'autres pays avec le même reproche qu'en Allemagne. Lorsque les cours se terminent tôt dans la journée, les élèves se trouvent souvent désœuvrés après les cours. C'est une source d'inégalité scolaire puisque les enfants de milieu favorisé peuvent suivre des activités extrascolaires favorables à leur scolarité. En Espagne, les parents regrettent que les élèvent soient livrés à eux-mêmes lorsque les cours ont lieu uniquement le matin (jornada continua) et le débat est récurrent dans les établissements entre les partisans de ce système et ceux qui prônent la jornada partida (les cours ont lieu matin et aprèsmidi). Les débats de nature différente peuvent se cristalliser sur l'augmentation ou 
la diminution du nombre d'heures d'instruction (Catalogne, Japon), sur la durée des vacances scolaires (Lituanie, Pologne), les rythmes scolaires hebdomadaires ( 5 à 6 jours d'étude hebdomadaire en Russie). Mais ces débats n'ont pas l'ampleur de celui mené en France.

Luc Chatel, dans sa lettre de mission au Comité de pilotage, place les travaux de réflexion et de redéfinition des rythmes scolaires sous le signe de la comparaison « Dans le cadre européen, l'observation des rythmes scolaires de pays comparables à la France permettra de discerner ce qui, dans leur conception, participe le cas échéant d'un meilleur succès des élèves ${ }^{4}{ }^{4}$

Le débat sur les rythmes scolaires n'est pas clos en France. Le rapport du Comité de pilotage ${ }^{5}$ paru le 4 juillet 2011 préconise dix mesures pour modifier les rythmes scolaires, et se termine par des graphiques reprenant les analyses comparatives d'Eurybase et de l'OCDE. On voit par là l'importance donnée à l'examen d'autres systèmes éducatifs pour la mise en œuvre d'une réforme des rythmes scolaires en France.

\section{NOTES}

1. Le Comité de pilotage est composé de seize membres, personnalités qualifiées sur les questions éducatives et de neuf parlementaires. Odile Quintin, ancienne directrice générale de l'Éducation et de la Culture à la commission européenne et Christian Forestier, administrateur général du Consevatoire national des arts et métiers, président le Comité.

2. Ces fiches, réalisées pour l'essentiel par le CIEP, sont consultables sur le site de la Conférence des rythmes scolaires dans la rubrique «Actualité internationale»: http://www.rythmesscolaires.fr/conference/comprendre-8/.

3. L'école à temps plein prend en charge les élèves toute la journée et tous les jours de la semaine. On trouvera dans le dossier d'actualité de la VST rédigé par Agnès Cavet (2011), «Les rythmes scolaires: pour une nouvelle dynamique des temps éducatifs ", une présentation détaillée des réformes menées en Allemagne.

4. In Conférence nationale sur les rythmes scolaires : « Rapport de synthèse des auditions, des débats en académie et des échanges sur Internet - janvier $2011 »$ p. 123.

5. Conférence nationale sur les rythmes scolaires, «Des rythmes plus équilibrés pour la réussite de tous : rapport d'orientation sur les rythmes scolaires », juin 2011. 
INDEX

Mots-clés : aménagement du temps scolaire, rythmes scolaires

Keywords : organisation of school time, school time

Palabras claves : organización del tiempo escolar, tiempo escolar

\section{AUTEUR}

BERNADETTE PLUMELLE

Responsable du centre de ressources et d'ingénierie documentaires du CIEP. 